\title{
Agôn
}

Revue des arts de la scène

Critiques | Saison 2014-2015

\section{Angelica Liddell, You Are My Destiny (Lo stupro di Lucrezia)}

Tirez sur la sorcière

\section{Marion Siéfert}

\section{(2) OpenEdition}

\section{Journals}

Édition électronique

URL : http://journals.openedition.org/agon/3166

DOI : $10.4000 /$ agon.3166

ISSN : 1961-8581

Éditeur

Association Agôn

\section{Référence électronique}

Marion Siéfert, "Angelica Liddell, You Are My Destiny (Lo stupro di Lucrezia) », Agôn [En ligne], Critiques, mis en ligne le 10 mars 2015, consulté le 23 septembre 2020. URL : http://journals.openedition.org/ agon/3166; DOI : https://doi.org/10.4000/agon.3166

\section{Ce document a été généré automatiquement le 23 septembre 2020}

Association Agôn et les auteurs des articles 


\section{Angelica Liddell, You Are My Destiny (Lo stupro di Lucrezia)}

Tirez sur la sorcière

\section{Marion Siéfert}

\section{RÉFÉRENCE}

Angélica Liddell, You Are My Destiny (Lo stupro di Lucrezia)

Théâtre de l'Odéon, Paris, décembre 2014

« Scandaliser est un droit. Être scandalisé est un plaisir. Celui qui refuse le plaisir d'être scandalisé

est un moralisateur. »

Pier Paolo Pasolini, dernière interview, 31 octobre 1975

Depuis La Casa de la Fuerza, découverte de l'édition 2010 du Festival d'Avignon, ravivée par Todo el cielo sobre la tierra en 2012, nombreux sont ceux qui se déclaraient inconditionnels d'Angelica Liddell, de sa puissance émotionnelle et de sa capacité à performer sur scène les textes qu'elle écrit. Son dernier spectacle, You Are My Destiny (Lo stupro di Lucrecia), présenté dans le cadre cossu du Théâtre de l'Odéon en décembre dernier, déçoit cependant ses fans les plus $\operatorname{ardents}^{1}$ et est reçu par un concert d'indignation, de réprobations et d'incompréhensions ${ }^{2}$. La plupart des critiques semblent être pris dans un paradoxe, accusant la metteure en scène tout à la fois de se répéter et de ne pas exécuter ce pour quoi ils l'aiment d'ordinaire. Étrange façon d'aimer une artiste que celle qui embrasse en étouffant et applaudit en contrôlant. Il y en aurait à la fois trop et pas assez dans You Are My Destiny. Trop de symboles, pas assez de texte; trop de bruit, pas assez de sens; trop de scènes étirées, pas assez de conclusions ; trop d'hommes, pas assez de femmes ; trop de chaos, pas assez de clarté ; trop d'excès, pas assez de mesure; trop de paix, pas assez de violence; et trop de violence pour pas assez de moments de grâce ${ }^{3}$. Sans aucune forme de procès, on a tiré sur la sorcière. 


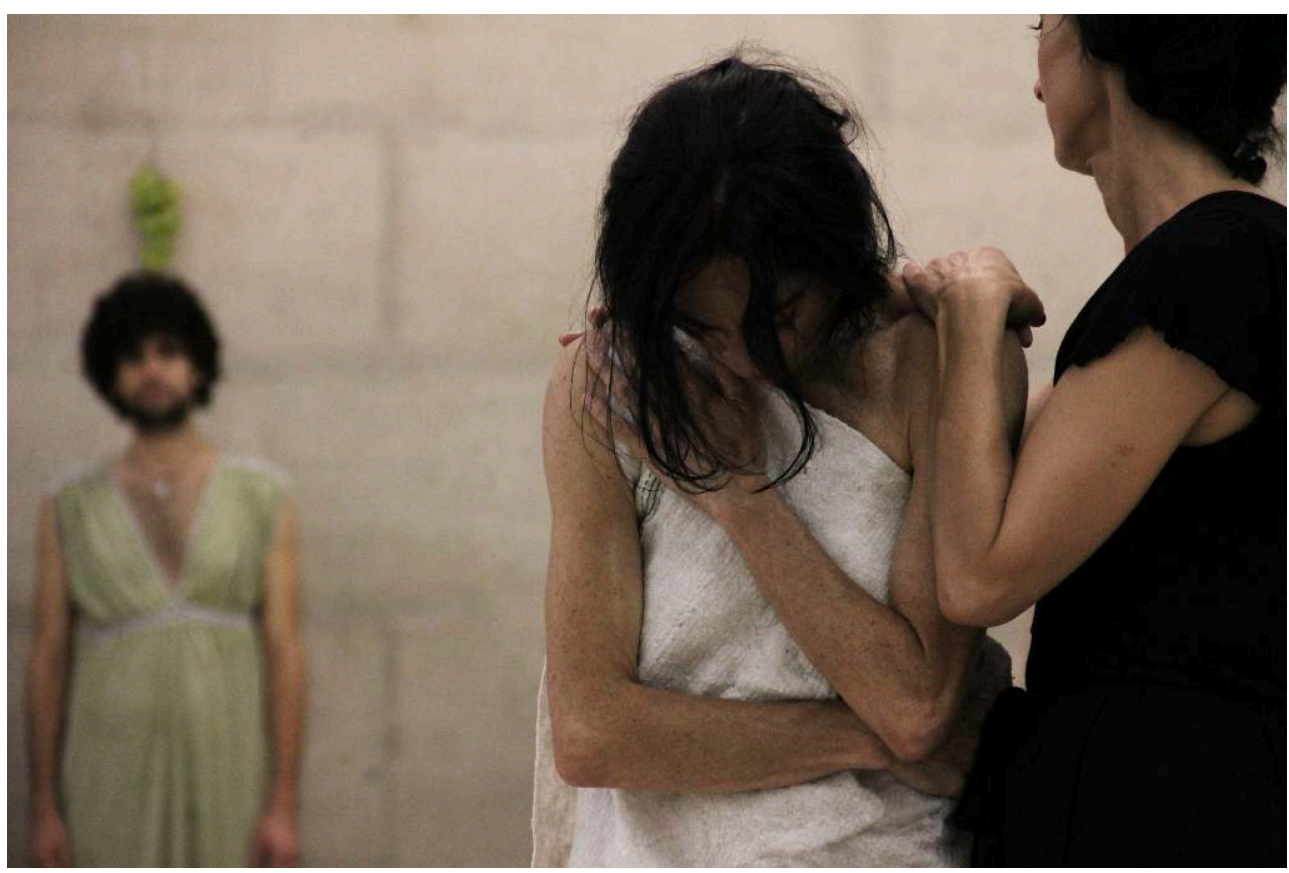

(c) Antonio Pina

\section{Le révélateur}

2 Selon la plupart des critiques, You Are My Destiny ne nous ferait vivre rien d'autre qu'un ennui profond, conséquence inévitable d'une complaisance trop grande de l'artiste pour sa propre matière ${ }^{4}$. À bien les lire, ils ne se sont pas seulement ennuyés : ils ont été insupportés par une durée dont ils n'ont su que faire. Pourtant, La Casa de la Fuerza et Todo el cielo sobre la tierra déployaient déjà une temporalité étirée, que les critiques avaient alors acceptée, et proposaient aux spectateurs de vivre une expérience radicale de la durée. Une durée qui ne pourrait s'appréhender comme une linéarité correctement organisée, au tempo adéquat et aux enchaînements agréables. Taillé dans une roche aux strates souterraines et multiples, le temps de You Are My Destiny prend les dimensions et la mesure d'une épreuve. Épreuve relevée comme un défi d'enfants par ses douze comédiens, qui battent le tambour de toute leur force. Épreuve de la répétition pour Lola Jiménez, son double muet sur scène, corps nu livré aux mains avides des hommes. Épreuve à laquelle Angelica Liddell se soumet elle-même lorsque, allant aux limites de la suffocation, elle va jusqu'à boire plusieurs litres de bière d'une seule traite.

3 Si Liddell a toujours été une artiste du temps, comment cela se fait-il qu'on lui reproche aujourd'hui une durée que l'on a jadis adorée ? Il y a bien une différence entre You Are My Destiny et les spectacles précédents qui avaient recueilli les faveurs des critiques : cette fois-ci, la metteure en scène se refuse à trancher dans le sens, à être univoque et à délivrer un propos avec son mode d'emploi. Dans ce spectacle, les scènes ne se résolvent pas sur une tirade de texte, les blocs de temps que l'artiste dispose ne permettent pas qu'une signification immédiatement saisissable se dégage du spectacle. $\mathrm{Au}$ contraire, les images déployées semblent se contredire ou s'étouffer les unes les 
autres; les scènes se succèdent plus qu'elles ne s'enchaînent; tambours, cris, danses et chants remplacent le discours articulé et la parole, d'ordinaire si centrale chez Liddell. Ne trouvant pas le texte attendu, les critiques font la découverte d'un temps brut, sans dialogue, sans monologue, sans "théâtre »: ils se retrouvent face à une simple durée. Leur temps idéal, ils le voudraient finalisé et homogène, comme un milieu neutre et stable, propice à accueillir un enchaînement d'événements et d'actions articulés les uns aux autres, justifiés par une idée globale et assemblés par une unité d'ensemble. Un temps auquel ils pourraient se fier et qui, au fond, ne les affecterait pas. Moins temps qu'espace, il en aurait la permanence, l'aptitude à être découpé en parties aisément situables et en tronçons de sens, sans pour autant perdre de sa continuité.

4 C'est s'empêcher de voir que chez Angelica Liddell, le temps agit comme révélateur. Il est cette durée nécessaire au vécu, à l'émergence des souvenirs de ses spectateurs, au surgissement, inopiné comme un précipité chimique, de la vérité du corps de ses performers. Ni trivial, ni fictif, le temps a des conséquences importantes pour les comédiens et les spectateurs, entraînés dans You Are My Destiny. Ce sont d'abord les douze acteurs qui, le dos appuyé contre la façade dorée du Palais des doges, tiennent la position de la chaise pendant une durée suffisante à les faire trembler, supplier et gémir de douleur. Et lorsque ces corps massifs et solides se craquèlent, c'est pour laisser éclater une faiblesse que leur force dissimulait : la vulnérabilité de Tarquin, le violeur de Lucrèce. La vérité que cherche à faire advenir Liddell n'est pas de l'ordre du dicible ou de la logique, mais est engendrée par l'endurance impitoyable des corps. C'est par le temps que quelque chose peut s'obtenir: non par la force, mais au contraire, par son épuisement. C'est seulement en confrontant la force à ses propres limites, en la poussant dans ses plus ultimes retranchements avec les armes mêmes de la performance, que la virilité peut alors apparaître comme ce qu'elle est, un masque qui dissimule une faiblesse.

5 C'est aussi le temps des spectateurs qui est creusé, dilaté, rattrapé par d'autres souvenirs, traversé par des courants plus anciens, agité par des remous profonds qui continuent à agir, bien après la représentation. Les réactions dans la salle sont éclatées, individuelles, traversées par un état accru de présence : c'est le rire solitaire d'une femme qui semble ne jamais vouloir s'arrêter, les pleurs silencieux d'un homme, le sursaut courroucé de plusieurs spectateurs qui quittent la salle à différents moments, la tranquillité placide et concentrée d'yeux qui contemplent, observent et parleront plus tard. Liddell est bien cette artiste du temps qui agit contre nos habitudes rythmiques, nos cadences dramatiques et taille dans la durée pour faire surgir d'autres temporalités propres à chacun, plus intimes et secrètes, refoulées ou tout simplement oubliées. Son temps rejoint celui des rêves et des réminiscences, il se mêle à des choses vues, lues et incorporées : il synthétise du vécu.

\section{L'inactuelle}

6 Avec You Are My Destiny, Angelica Liddell interprète l'histoire de Lucrèce de manière non-orthodoxe. Déjà narré notamment par Tite-Live et Shakespeare, ce récit fait de la jeune femme violée par Tarquin - le fils du roi de Rome - un symbole de vertu féminine. Comme le raconte l'histoire, Lucrèce se suicide devant son père et son mari après leur avoir révélé l'outrage dont elle a été la victime, sauve ainsi son honneur et celui de ses proches, précipite la chute de la tyrannie et accélère l'avènement de la 
République. Angelica Liddell s'inscrit contre l'histoire officielle, reprise et confortée dans sa légitimité par certains articles ${ }^{5}$, et fait la guerre à tous ceux qui voudraient assigner et contenir Lucrèce - et par extension, toutes les femmes - à une bienséance paralysante, à une vertu digne, raisonnable et mortifère. C'est cette Lucrèce historique, triste, muette et stoïque - interprétée par Lola Jiménez - qui attire les insultes, le crachat et le mépris d'Angelica Liddell. Une Lucrèce qui voit son sort définitivement scellé par le viol et envisage le suicide comme seule planche de salut. Ce qui révolte la metteure en scène, c'est cette "vision trop plate des choses " qui, d'une part, fait de l'histoire de Lucrèce « un symbole de la chute des tyrans en reléguant le viol au second plan ", et d'autre part, la cantonne à un " point de vue strictement féministe " ${ }^{6}$. Ce qui l'insupporte, c'est cette façon dont l'histoire ne retient que les grandes significations et gomme les petites violences : celles du mari, qui réduit Lucrèce à un vulgaire objet de pari, et celles de cette société de matrones vertueuses, qui considère qu'une femme ne peut survivre au viol. Dans You Are My Destiny, Liddell change donc radicalement la perspective traditionnelle, qui voudrait faire de Lucrèce soit la figure salvatrice et vertueuse d'une lutte politique, soit la victime passive de la brutalité masculine. Elle répond à ces assignations à la vertu, en s'intéressant au point de vue que ces interprétations dominantes occultent : celui de Tarquin et donc, celui très dérangeant, de son désir.

7 Le désir est chose trouble, complexe, injuste et irrémédiablement amorale. Il en révèle bien plus sur les contradictions propres à chacun, sur ces points intimes où se jouent les dilemmes politiques, moraux et personnels, que tous les principes et les conduites de vie arrêtés. C'est lui qui remet en mouvement les certitudes rassurantes car établies une fois pour toutes comme justes et vraies. Non, Liddell ne légitime pas Tarquin, mais fait l'hypothèse que le violeur nous en dit peut-être autant sur le viol que la victime. C'est par sa manière de s'emparer d'un récit construit et entériné par 2000 ans d'histoire occidentale que Liddell est inactuelle : elle s'inscrit contre la canonisation d'une histoire exemplaire à laquelle on reconnaît une éternelle actualité et contre un féminisme rectiligne et correct, adéquat à une morale qui se prétend universelle et intouchable. Pour Liddell, l'histoire n'est ni une poussière d'événements, ni la compréhension de leur logique, elle devient son propre drame, sa propre tragédie, sa propre sorcellerie.

8 Il y a chez Angelica Liddell la volonté d'assumer totalement les événements de son existence en les accueillant comme son propre destin. Transformant le hasard en méthode, elle refuse de réduire les rencontres et les aléas de la vie à de simples anecdotes, mais reconnait en eux sa propre nécessité, en dépit même de ses principes ou de ses convictions antérieures. À la Biennale de Venise, elle tombe ainsi sur douze comédiens italiens qu'elle apprend à connaître au cours d'un workshop et sur trois chanteurs ukrainiens qui chantaient dans la rue. Ce seront eux qui donneront la direction au spectacle et feront de You Are My Destiny l'« envers rédempteur $»^{7}$ de $L a$ Casa de la Fuerza, avec lequel il forme un diptyque. Alors que Venise était depuis La Casa de la Fuerza la ville de l'humiliation, de la haine et de la vengeance de Liddell à l'encontre des hommes, elle devient l'endroit où peut se vivre une collaboration généreuse avec eux. Dans You Are My Destiny, Angelica Liddell synthétise dans un même mouvement un récit façonné par des millénaires d'histoire humaine et des rencontres qui se sont imposées à elle avec la force des révélations. Sa création ne découle ni d'une interprétation délibérément provocante de l'histoire de Lucrèce, ni d'une complaisance 
narcissique : elle travaille à partir des difficultés concrètes de sa propre existence. Le mythe et la vie se mêlent et c'est leur rencontre explosive et contradictoire que Liddell met en scène.

9 Dans les reproches qu'ils adressent à You Are My Destiny, les critiques opèrent par réduction : ils réduisent le spectacle à un pitch provocateur (Liddell transforme un viol en une histoire d'amour) et balaient ainsi d'un revers de main l'ensemble de son œuvre précédente qui, de Mais comme elle ne pourrissait pas... Blanche-Neige à La Casa de la Fuerza, traitait avec une profondeur et une finesse rarement rencontrées la question du viol. Peut-on honnêtement croire que la metteure en scène aurait aussi promptement retourné sa veste pour défendre cette fois-ci le violeur? C'est pourtant la thèse que nombre de critiques semblent soutenir. Tous la rappellent à l'ordre et condamnent sévèrement son manque de morale et son inconscience politique ${ }^{8}$. Angelica Liddell «a orchestré une toxique variation qui condamne et légitime à la fois le violeur, jusqu'à défier la femme violée d'aimer son bourreau, le seul peut-être à la tant désirer ", déclare Fabienne Pascaud. Corinne Denailles insiste : « Elle explique comment elle a fini par aimer le bourreau qui, à travers le viol, commet un ultime geste d'amour. » Et Annie Chénieux renchérit: "Dans sa dernière création, elle absout le violeur de Lucrèce." Tandis que Frédérique Villemur redresse la barre de l'histoire: "Angélica Liddell renverse donc les lignes de l'histoire de Lucrèce rapportée par Tite-Live, et traduite en un long poème par Shakespeare, pour transformer la haine née de l'humiliation, et retourner la vengeance en un cri d'amour. »

Inconsciente dans sa folie provocatrice, empêtrée dans ses propres souffrances et dans un dualisme judéo-chrétien, la metteure en scène défendrait l'indéfendable et transformerait la violence et l'humiliation du viol en un cri d'amour qui absoudrait le violeur. Il y aurait, dans ce choix du désir, un déni de la part d'Angelica Liddell qui oblitérerait les implications politiques du viol, faisant comme si le corps n'était pas structuré historiquement et socialement, ce qui rendrait insoutenable la façon dont elle s'intéresse aux faiblesses du bourreau et donc, à sa part d'humanité 9 . Avant de comprendre ce qui fait réellement scandale dans You Are My Destiny, répondons brièvement à cette dernière accusation, en citant Liddell dans un entretien réalisé à l'occasion des représentations de La Casa de la Fuerza à l'Odéon : "Dans Mais comme elle ne pourrissait pas... Blanche-Neige, mon intention était de parler des enfants soldats, de leur rôle dans les guerres, de l'utilisation des petites filles, de la fonction qui leur est assignée parce qu'elles sont des filles : butin de guerre, violées, objet de chantage ou de châtiment." Ainsi, plutôt que de voir dans You Are My Destiny, soit une répétition ennuyeuse d'images et de motifs déjà présents dans ses précédentes pièces, soit un volte-face provocateur par rapport aux idées qu'Angelica Liddell était supposée défendre, il faut plutôt y lire un approfondissement d'une réflexion qui prend racine dans le cœur même de son travail et qui court le risque de la contredire, la creuser, de l'enrichir et de la déplacer, de se retenir d'être définitivement pour ou contre en multipliant les regards, manière de redonner au regard son innocence (qu'il faut distinguer de la naïveté) ${ }^{10}$. Angelica Liddell s'emploie à complexifier l'histoire en rendant visible les forces qui s'y opposent et, ainsi, en organisant leur hiérarchie : il ne s'agit pas seulement de s'engager pour la figure souillée, vertueuse et salvatrice de Lucrèce, mais de lui opposer un personnage antithétique, la sorcière; de reconnaître quelle sorte de force s'exprime chez l'une et chez l'autre; de dire certes non à l'une et oui à l'autre, mais de les montrer ensemble. 
11 Si You Are My Destiny scandalise, c'est par son renversement des valeurs ${ }^{11}$, par sa manière de ne pas tenir compte d'une morale simple et prétendument universelle, en réinvestissant un récit historique particulier afin d'en interroger les antinomies que l'histoire a figées (le désir du violeur vs la souillure de la femme victime; le crime déshonorant vs le suicide vertueux). Il s'agit de montrer ce que cherche à dissimuler le monde de la culture dans les discours appris, les engagements politiques et les indignations réflexes. En mettant son nez dans ce qu'elle appelle le «puits d'excréments de l'âme humaine ", Angelica Liddell choisit d'habiter la contradiction.

\section{La sorcière}

« Nous vivons de manière beaucoup trop propre, alors que nos désirs sont faits de boue. J'écris mes spectacles à partir de cette boue, pas du jugement. L'art n'est pas la loi, ni une organisation d'assistantes sociales. C'est un acte d'épiphanie individuelle. Trop de gens confondent aujourd'hui l'expression et la correction. " Angelica Liddell

Face à toutes les contradictions qui configurent You Are My Destiny et qui en font sa force explosive et libératrice, les critiques n'ont exprimé qu'une incompréhension réprobatrice. Selon eux, le spectacle voudrait réunir ce qui est incompatible et ne pourrait être le fruit que d'un «corps malade débordé par le mouvement de ses organes : saturé, obsédant, répétitif, gargouillant, à la fois immobile et en perpétuel mouvement exagéré ${ }^{12}$. Confus car dégénérescent, chaotique car déréglé, You Are My Destiny serait la sécrétion mal contrôlée d'un corps incontinent et excessif, celui d'Angelica Liddell. Ce qu'ils lui reprochent, en des termes qui relèvent plus de la condescendance et de l'insulte que de la critique, c'est au fond d'être contradictoire. Or, c'est justement là que se joue tout le travail de Liddell. Toujours elle cherche à dépasser l'évaluation unilatérale, hémiplégique, d'un des termes en présence et redonne un pouvoir de pensée aux ambivalences. Dans la réduction opérée par ces critiques de l'art au pathologique, de l'artiste à la femme, se trouve le rouage vicié de leur argumentation. Parce qu'ils ne peuvent souffrir les contradictions et donc, une pensée qui dérange et bouscule les découpages moraux les plus intimes, ils la déclassent et la maintiennent en-deçà du champ de l'art.

De cette incompréhension face à ce qui apparaît comme un propos brouillon et vide de sens surgit le rejet massif de ce qu'Angelica Liddell incarne sur scène : une sorcière, puissance qui trouble l'ordre de la mise en scène, brouille les cartes des grands principes moraux et des catégories logiques et esthétiques, et s'épanouit dans un fouillis chaotique, mal-élevé et un peu sale ${ }^{13}$. Le vocabulaire qui désigne " la Liddell » est éloquent, tant il fait ressurgir les fantasmes les plus troubles qui entourent la femme dangereuse, mi folle mi nuisible, scandaleuse et obscène, parce que mettant à mal un ordre. «Déesse noire ", " grande prêtresse ${ }^{14}$, Angelica Liddell " se vautre » ${ }^{15}$, "se gave", " engloutit les bouteilles de bière à s'en rompre le cou ", " piétine des grappes de raisin déposées au sol comme des bouts de chair, nue dans un sacrifice expiatoire, orgiaque jusqu'à l'ivresse masturbatoire $»^{16}$. Elle ne parle pas, mais 
"vocifère $~^{17}$ et laisse sortir des «éructations obscures " ${ }^{18}$ qui provoquent "l'écœurement ${ }^{19}$, "se badigeonne le corps de n'importe quoi, se tire brutalement les seins, ou se masturbe presque avec une bouteille de bière $»^{20}$, martyrise ses comédiens, "les fait souffrir pour de vrai, leur inflige de véritables tortures ${ }^{21}$. C'est donc un véritable lynchage, une humiliation pour débauche, licence et atteinte aux bonnes mœurs qui est fait à Liddell. D'où la multiplication des rappels à l'ordre dans les articles, où certains critiques, brandissant leur petit bâton de censeur, ne cessent d'appeler au "bon goût ", à la " tempérance "22, allant même, comme Stéphane Capron, jusqu'à conclure son article par un climax sexiste, en recommandant à ses lecteurs de s'intéresser à d'autres "femmes metteuses en scène [...] dont les images et les réflexions nous parlent beaucoup plus [...] avec de vrais discours beaucoup plus construits. $»^{23}$ Refusant de décrire les images et les situations d'une pièce dans laquelle ils ne trouvent aucun sens, ils délirent le spectacle et parlent de choses qui n'existent pas. Des grappes de raisin deviennent ainsi des «bouts de chair ", des éclaboussures sont transformées en " postillons d'eau sale ${ }^{24}$, ils fantasment des masturbations et des orgies sur scène, attribuent des actions de Lola Jiménez à Angelica Liddell, ne font plus la distinction entre la metteure en scène et Lucrèce. Le contresens est énorme : on reproche son manque de mesure et ses entorses au bon goût à un spectacle qui ne cesse justement de se rebeller contre l'assignation à la bienséance, contre tout ce qui dans un champ esthétique et moral, voudrait contraindre les femmes à une idée étroite et restrictive de la vertu. La faute d'Angelica Liddell est de ne pas tenir son rang.

\section{La pensée sauvage}

14 Certains spectacles nous font penser pendant la représentation, ils se débrouillent pour que notre intellect soit toujours en état d'éveil et disposent, dans un fin et intelligent désordre, les éléments qui nous permettront de comprendre; leur art résulte du montage et de l'analyse. D'autres nous font penser après : pendant la représentation, c'est alors notre mémoire qui est sollicitée, si bien que l'on se souvient tout à coup d'événements oubliés, comme des bribes de rêves qui remonteraient à la surface ; leur art relève de la synthèse, ils nous reconfigurent. You Are My Destiny appartient plutôt à la deuxième catégorie : s'ouvrant sur le récit d'un rêve, il se situe sur un terrain où les distinctions entre fantasme, réalité psychique et réel seraient suspendues ${ }^{25}$. Accoudée à un sofa, Angelica Liddell raconte à Lola Jiménez comment, dans une rue sombre de Venise, elle se met à suivre un inconnu, certaine qu'il était celui qui allait la violer puis l'assassiner. Le rêve se poursuit, elle perd la trace de son assassin et se retrouve dans une mercerie remplie de bonnes femmes qui se jugent à coups de talents ménagers, se surveillent mutuellement, ne cessent de se rappeler à l'ordre. Dans cette atmosphère nauséabonde qui "pue la menstruation", Liddell recherche désespérément son inconnu, sans parvenir à le retrouver. Lancer You Are My Destiny sur le récit d'un rêve, c'est donner une clé de lecture aux spectateurs: le spectacle s'ancre dans l'ordre du fantasme. Il ne faut donc y chercher ni description d'une réalité objective, ni affirmation d'une vérité historique, explicite et universelle, mais y suivre les interrogations d'une géologue sur les pulsions inavouables qui peuvent nous structurer, sur des peurs que nous ne parvenons pas à formuler à nous-mêmes, sur la face sombre de nos désirs. 
15 La sauvagerie de la pensée de Liddell tient à sa capacité à libérer des affects extrêmement puissants et à fracasser les préceptes moraux qui soutiennent l'édifice social. Son théâtre pourrait s'apparenter au délire ou au nihilisme, s'il n'était conçu comme un rituel, que Liddell orchestre à vue, donnant ses ordres et indications par signes et gestes. Sur scène, rien n'est laissé au hasard; tout semble obéir à une codification stricte et précise, traversée cependant par un plaisir du jeu et une énergie anarchique. Chaque action est investie par un refus radical de toute simulation, cette « imitation dérisoire de la réalité » dont parlait Artaud. Chaque événement obéit à la nécessité d'exercer une efficacité symbolique ou sociale. Avec toute la puissance que lui donne le rituel, Liddell exhume des impensés, traque les instincts constitutifs de notre société et retrouve les origines immorales de notre morale, illogiques de notre logique, infondées de nos définitions. Dans You Are My Destiny, on voit des enfants que l'on s'apprête à tuer afin d'en faire des hommes ; une femme (Lola Jiménez) murée dans un silence mortifère ; un couple que l'on marie en même temps qu'on le prépare à la mort. Et lorsque Liddell lave les pieds des hommes qu'elle a soumis au supplice de la chaise, on perçoit la pulsion sadique qui travaille le sentiment de compassion, ce ferment de l'ordre social qui apaise et calme les révoltes. Ne s'arrêtant jamais à la signification définitive d'une scène, elle change soudainement la perspective, passant d'une scène de consolation à l'exhibition brutale de l'instinct qui la structure. Le travail de Liddell ne peut se résumer à une stratégie de provocation car son intention première n'est pas de choquer. En revanche, elle scandalise car elle dynamite nos certitudes morales et travaille à rendre visible toutes ces fois où notre pensée se fige en des principes rigides, et perd ainsi de son efficacité et de son aptitude à transformer le réel.

Si You are My Destiny est profondément libérateur, c'est qu'il ne détruit pas pour avoir le plaisir cynique de danser sur les ruines. Au contraire, Angelica Liddell propose une autre voie, certes intime et personnelle, en investissant un personnage, celui de la sorcière. Grimaçante, tantôt hideuse, tantôt comique, elle trouve une joie profonde et drôle, à faire tous ces gestes honteux, proscrits de l'espace bien élevé de la culture. Le spectacle commençait sur le rêve d'un fantasme de viol et se termine par une danse clownesque du sexe féminin. Dans cet épilogue qui semble improvisé, Angelica Liddell enlève sa culotte, met des mauvais tubes italiens à pleine pompe, assume un mauvais goût outrancier et parodie une scène de peep-show, lieu par excellence de l'exhibition $\mathrm{du}$ sexe féminin. Mais cette fois-ci, il n'est ni pornographique, ni obscène, ni mystérieux, ni érotique. Angelica Liddell ne le sublime pas, elle n'organise pas son apparition par un savant jeu d'éclairages qui rappellerait les toiles des maîtres ${ }^{26}$, elle ne le dégrade pas, ne le fait pas souffrir, mais le rend joyeux, comique, plein d'humour. Son sexe semble se moquer des gens qui le regardent depuis tant d'années comme un obscur objet de désir. Il rigole et affirme son pouvoir sur la représentation. La sorcière jubile. Elle a envoyé valdinguer pour un temps la princesse, son père, sa mère, son mari, son frère et ses sœurs, les bonnes femmes qui la contrôlaient et les ordures qui voulaient la brûler. Elle scandalise et s'en amuse, et roule une grosse pelle à la morale. 


\section{NOTES}

1. Alors qu'Armelle Héliot du Figaro proclame qu' "Angelica Liddell s'égare au théâtre de l'Odéon », Anna Sigalevitch (France Culture) se demande « ce qu'il est devenu d'Angelica Liddell » et Frédérique Villemur (Agôn) regrette « l'intensité des dernières pièces ». Tous, dans l'ensemble, clament leur déception: "Déception, on reste sur ce mot», conclut Arnaud Laporte dans l'émission « La Dispute».

2. Il n'y a guère que Fabienne Darge (Le Monde) et Jean-Paul Thibaudat (Rue 89) qui défendent le spectacle.

3. Tous regrettent l'absence de texte et donc de sens. Ainsi, Anna Sigalevitch dans l'émission « La Dispute » : «Le sens va arriver, le sens va arriver [...]. J'étais très concentrée. [Liddell] prend le micro, puis en fait, elle ne dit rien. » D'autres, comme Arnaud Laporte, lui reprochent de ne pas se faire autant souffrir que dans les autres spectacles et notamment, de ne pas boire suffisamment de bière pour être vraiment ivre.

4. "Mais là, on s'est royalement ennuyé", annonce Stéphane Capron (sceneweb). Et plus loin : «L'ensemble du spectacle est long, pesant, insupportable ». " Il ne se passe rien, ça dure, ça dure... ça dure trop - comme si la souffrance physique de ces hommes devait attiser l'impatience du spectateur ", se plaint Philippe Lançon (Libération), qui compare le spectacle à une "messe, longue comme celle de Pâques». Fabienne Pascaud (Télérama) parle de situations "étirées jusqu'à l'insupportable ", Corinne Denailles (webthéâtre) répète à l'envie que "cela n'en finit pas » et Annie Chénieux (Journal du Dimanche) évoque l'« ennui », la « lassitude » de cette pièce où Liddell «étire la durée jusqu'à l'agacement ", tandis que Frédérique Villemur (Agôn) déclare $q^{\prime}$ '« on se lasse de longues séquences complaisamment provocantes", si bien que l'« on ne sait quel parti-pris sera tenu jusqu'au bout, si ce n'est celui d'une dissolution finale, où le fossoyeur l'attend».

5. Frédérique Villemur rappelle dans Agôn: «L'histoire est connue : il s'agit du viol de Lucrèce, par Sextus Tarquin, fils du roi de Rome. L'outrage devient exceptionnel par les conséquences qu'il entraîne : suicide de Lucrèce, renversement de la tyrannie et proclamation de la République. Mais de tout cela nous n'entendons rien ici. Car Angélica Liddell dans You are my destiny s'insurge contre la vertu du geste héroïque de Lucrèce. Elle se «révolte contre (sa) récupération politique », voulant "parler du désir, du pouvoir du sexe sur la volonté ; [son] intention était de comprendre Tarquin ». Voilà qui est dit. »

6. Entretien avec Angelica Liddell pour le Festival d'Automne à Paris. Propos recueillis et traduits par Christilla Vasserot.

7. Entretien avec Angelica Liddell pour le Festival d'Automne à Paris. Propos recueillis et traduits par Christilla Vasserot.

8. «Voici une pièce de théâtre qui dérangera plus d'une féministe, plus d'une femme, autant dire plus de la moitié de l'humanité, troublera certains hommes ", déclare Frédérique Villemur en ouverture de son article.

9. C'est l'argument développé par Frédérique Villemur : «Au cœur de cette irréductible pulsion dans le désir, elle confond violence et amour, refusant de prendre en charge la dimension politique de l'intime quand tout rapport de sexe implique un rapport de pouvoir. Angélica Liddell se situe en-dehors du temps de l'Histoire. Oubliant ce qui dans le rapport au corps relève d'une domination capitaliste et patriarcale, elle oblitère la dimension politique du viol, en particulier à une époque où il est mobilisé de manière systématique comme arme de guerre. Quand on pense aux femmes yézidies enlevées par les membres de Daech ou aux enfants nés des viols du génocide rwandais, comment soutenir ce propos : «J'ai voulu voir l'homme dans sa fragilité à l'instant où on le voit dans la situation du bourreau »?» 
10. Je défends ici l'idée que le travail de Liddell s'inscrit dans un perspectivisme, qu'il faut distinguer d'un relativisme. Le perspectivisme a pour première ambition de récuser le dogme d'objectivité. Il ne s'agit donc pas de défendre le règne de l'opinion, mais de reconnaitre que différentes positions s'opposent, se conjuguent et se hiérarchisent, et que le monde humain, avec ses valeurs, est le fruit de cette coexistence. Il y a l'idée que toute perspective est injuste, mais que si on apprend à les multiplier, on ouvre des voies à des manières de penser multiples et opposées et on rétablit nécessairement une forme de justice, c'est-à-dire de hiérarchie : oui, une multitude de perspectives existe, mais toutes ne se valent pas, en fonction des contextes et des critères invoqués. Je renvoie ici notamment à l'analyse de la préface d'Humain trop humain de Nietzsche que fait Monique Dixsaut dans son ouvrage Nietzsche. Par-delà les antinomies (p. 137-143). 11. C'est bien ce grand détournement qui constitue le socle du scandale pour Frédérique Villemur, qui insiste à de multiples reprises sur la manière dont Angelica Liddell " renverse ", « retourne » et « détourne».

12. Philippe Lançon, « Piqûre de Mystique » (Libération).

13. «Ce grand capharnaüm de sons, images et lumières », écrit Philippe Lançon (Libération).

14. Corinne Denailles, «Chaos » (webtheatre).

15. Frédérique Villemur, Marie-José Sirach et Philippe Lançon emploient ce terme.

16. Frédérique Villemur, « Angelica Liddell. De stupre et de sang » (Agôn).

17. Corinne Denailles, « Chaos » (webtheatre)

18. Fabienne Pascaud, «Entre douleur et plaisir, les tortures scéniques d'Angelica Liddell » (Télérama).

19. Stéphane Capron, « La torture amoureuse d'Angelica Liddell » (Sceneweb).

20. Fabienne Pascaud.

21. Corinne Denailles.

22. "Aucune échappatoire de bon goût, de tempérance n'est laissée au spectateur ", se plaint Philippe Lançon, tandis que Stéphane Capron condamne: «La folie destructrice de Lucrèce se matérialise par des scènes de tortures indisposantes. »

23. Stéphane Capron, « La torture amoureuse d'Angelica Liddell » (Sceneweb).

24. Successivement Frédérique Villemur et Philippe Lançon.

25. «Un joyau sous le désastre», entretien avec Anne Dufourmantelle, psychanalyste et philosophe, pour le Théâtre de l'Odéon. http://www.theatre-odeon.eu/en/le-magazine/2014/10/ angelica-liddell-un-theatre-de-la-merveille

26. C'est notamment ce qu'on pouvait voir dans Le verrou (figure de fantaisie attribuée à tort à Fragonard), spectacle de Gaëlle Bourges, où le dévoilement du sexe féminin était l'occasion d'un exercice de libertinage - certes masqué par tout un discours théorique surplombant. 